\title{
Article
}

\section{Physics of Eclipsing Binaries: Modelling in the new era of ultra-high precision photometry}

Pavlovski, K., Bloemen, S., Degroote, P., Conroy, K., Hambleton, Kelly, Giammarco, J.M., Pablo, H., Prša, A., Tkachenko, A. and Torres, G.

Available at http://clok.uclan.ac.uk/14588/

Pavlovski, K., Bloemen, S., Degroote, P., Conroy, K., Hambleton, Kelly, Giammarco, J.M., Pablo, H., Prša, A., Tkachenko, A. et al (2013) Physics of Eclipsing Binaries: Modelling in the new era of ultra-high precision photometry. EAS Publications Series, 64 . pp. 269-276. ISSN 1633-4760

It is advisable to refer to the publisher's version if you intend to cite from the work. http://dx.doi.org/10.1051/eas/1364037

For more information about UCLan's research in this area go to http://www.uclan.ac.uk/researchgroups/ and search for <name of research Group>.

For information about Research generally at UCLan please go to http://www.uclan.ac.uk/research/

All outputs in CLoK are protected by Intellectual Property Rights law, including Copyright law. Copyright, IPR and Moral Rights for the works on this site are retained by the individual authors and/or other copyright owners. Terms and conditions for use of this material are defined in the policies page. 


\title{
PHYSICS OF ECLIPSING BINARIES: MODELLING IN THE NEW ERA OF ULTRA-HIGH PRECISION PHOTOMETRY
}

\author{
S. Bloemen ${ }^{1,2}$, P. Degroote ${ }^{2}$, K. Conroy ${ }^{3}$, K.M. Hambleton ${ }^{4,5}$, \\ J.M. Giammarco ${ }^{6}$, H. Pablo ${ }^{7}$ and A. Prša ${ }^{5}$
}

\begin{abstract}
Recent ultra-high precision observations of eclipsing binaries, especially data acquired by the Kepler satellite, have made accurate light curve modelling increasingly challenging but also more rewarding. In this contribution, we discuss low-amplitude signals in light curves that can now be used to derive physical information about eclipsing binaries but that were unaccessible before the Kepler era. A notable example is the detection of Doppler beaming, which leads to an increase in flux when a star moves towards the satellite and a decrease in flux when it moves away. Similarly, Rømer delays, or light travel time effects, also have to taken into account when modelling the supreme quality data that is now available. The detection of offsets between primary and secondary eclipse phases in binaries with extreme mass ratios, and the observation of Rømer delays in the signals of pulsators in binary stars, have allowed us to determine the orbits of several binaries without the need for spectroscopy. A third example of a smallscale effect that has to be taken into account when modelling specific binary systems, are lensing effects. A new binary light curve modelling code, PHOEBE 2.0, that takes all these effect into account is currently being developed.
\end{abstract}

${ }^{1}$ Dept. of Astrophysics, IMAPP, Radboud University Nijmegen, PO Box 9010, 6500 GL, Nijmegen, The Netherlands

${ }^{2}$ Instituut voor Sterrenkunde, KU Leuven, Celestijnenlaan 200D, 3001 Heverlee, Belgium

3 Dept. of Physics and Astronomy, Vanderbilt University, Nashville, TN 37235, USA

4 Jeremiah Horrocks Institute, University of Central Lancashire, PR1 2HE, UK

5 Dept. of Astrophysics and Planetary Science, Villanova University, 800 Lancaster Ave., Villanova, PA 19085, USA

6 Dept. of Astronomy and Physics, Eastern University, Saint Davids, Wayne, PA 19087, USA

7 Dépt. de Physique, Université de Montréal, CP 6128, Succursale Centre-Ville, Montréal, QC, H3C 3J7, Canada 


\section{Introduction}

In recent years, satellites such as Kepler (Borucki et al. 2010) and CoRoT (Auvergne et al. 2009) have performed photometric observations of eclipsing binary stars at unprecedented precision and duty cycle. Of the roughly 190000 stars that the Kepler satellite has observed, more than 1 per cent is identified as an eclipsing binary. This results in a sample that is large enough to perform statistical studies (see the catalog papers Prša et al. 2011; Slawson et al. 2011; and Kirk et al. 2013, in prep.), while every individual light curve by itself allows one to study an individual target in unprecedented detail. Kepler light curves of eclipsing binaries show features that were not seen before, but now become apparent because of the high accuracy of the brightness measurements and the time span of the observations of up to 4 years for many of the targets. In this contribution, we discuss recent studies of Doppler beaming in Section 2. Next, we briefly discuss features that can be attributed to light travel time delays in Section 3 and the effect of gravitational lensing in Section 4.

\section{Doppler beaming}

When the first month of Kepler data was released at the end of 2009, two binaries were found that not only showed eclipses, ellipsoidal modulation and reflection effects, but also persistently showed a difference between the two ellipsoidal maxima that appear per orbital cycle. The reason for these differences turned out to be Doppler beaming, also known as (Doppler) boosting. Doppler beaming was first observed by Maxted et al. (2000) in ground based data, but at low significance. The first two binaries with clear Doppler beaming signals found in Kepler data were KPD $1946+4340$, a binary with an orbital period of $9.7 \mathrm{~h}$ consisting of a subdwarf B star and a white dwarf (Bloemen et al. 2011), and KOI-74, which has an orbital period of $5.2 \mathrm{~d}$ and consists of a main sequence A star and a low-mass white dwarf (van-Kerkwijk et al. 2010).

Doppler beaming is a combination of three effects that are all caused by the motion of a star relative to the observer:

1. Light gets beamed towards the direction of motion of the source. This causes a star to appear slightly brighter when it moves towards the observer in its binary orbit;

2. The photon arrival rate is enhanced when the star moves towards the observer. This also causes the star to appear brighter when it moves in the direction of the observer;

3. The Doppler shift of the spectrum relative to the bandpass of the instrument. Depending on the spectrum on the source, the observed flux will be slightly increased or decreased.

Prior to the launch of Kepler, Loeb \& Gaudi (2003) already discussed the effect for systems with planets and Zucker et al. (2007) discussed the case of binary stars, 
thereby predicting the discovery of "Beaming binaries". The beaming amplitude can be approximated, for radial velocities much lower than the speed of light, by

$$
F_{\lambda}=F_{0, \lambda}\left(1-B \frac{v_{r}}{c}\right),
$$

in which $F_{\lambda}$ is the observed flux and $F_{0, \lambda}$ the emitted flux (Loeb \& Gaudi 2003, notation as in Bloemen et al. 2011). $B$ is the so-called beaming factor, which includes the three effects described above: $B=5+\mathrm{d} \ln F_{\lambda} / \mathrm{d} \ln \lambda$. The beaming factor thus depends on the spectrum of the star and the wavelength of the observations. For broadband photometry, one has to use a photon weighted bandpass-integrated beaming factor

$$
\langle B\rangle=\frac{\int \epsilon_{\lambda} \lambda F_{\lambda} B d \lambda}{\int \epsilon_{\lambda} \lambda F_{\lambda} d \lambda}
$$

in which $\epsilon_{\lambda}$ is the response function bandpass.

If the light contribution of one star is negligible compared to the other's, as is the case for the two Kepler binaries in which the effect was discovered first, measuring the amplitude of the effect gives a direct photometric measurement of the radial velocity amplitude of the star. In both cases, spectroscopic measurements were in perfect agreement with the photometric values (Bloemen et al. 2011; Ehrenreich et al. 2011; Bloemen et al. 2012). The uncertainty on the photometric values is in both cases determined by the uncertainty on the spectrum-dependent beaming factor rather than by the measured beaming amplitude. One should be careful, though, when interpreting the Doppler beaming signal when a binary's components both contribute significantly to the total light. In that case, the Doppler beaming signals of both stars will (at least partly) cancel each other out.

As an example of what a light curve with Doppler beaming looks like and how it is built up, we show the light curve of KPD 1946+4340 in Figure 1, as presented in Bloemen et al. (2011). The green data points show the phasefolded light curve, and the black lines the model created using LCURVE Copperwheat et al. (2010). In the different panels, from top to bottom, the model is made more sophisticated by adding the effects of eclipses, reflection, ellipsoidal modulation, gravitational lensing and beaming.

As shown by Zucker et al. (2007), the amplitude of the Doppler beaming effect drops at a slower rate with increasing orbital period than reflection effects and ellipsoidal modulation. As such, the beaming effect will be the dominant effect in long-period binaries. Its detection in binaries with periods of tens of days or longer, though, is limited by our understanding of long-term instrumental trends in observed light curves and our ability to remove such trends without touching the astrophysical signal. The apparent mismatch between the expected and observed Doppler beaming signal in KIC 5006817 (Beck et al. 2013, submitted) is probably related to such a detrending issue.

In the case of a binary with a circular orbit, the signals of Doppler beaming, ellipsoidal modulation, and reflection can all be modelled with sine curves. Taking into account the right phase offsets between the three effects (beaming is maximum at phase 0.25 , ellipsoidal modulation at 0.25 and 0.75 and reflection at 0.5 ) and 


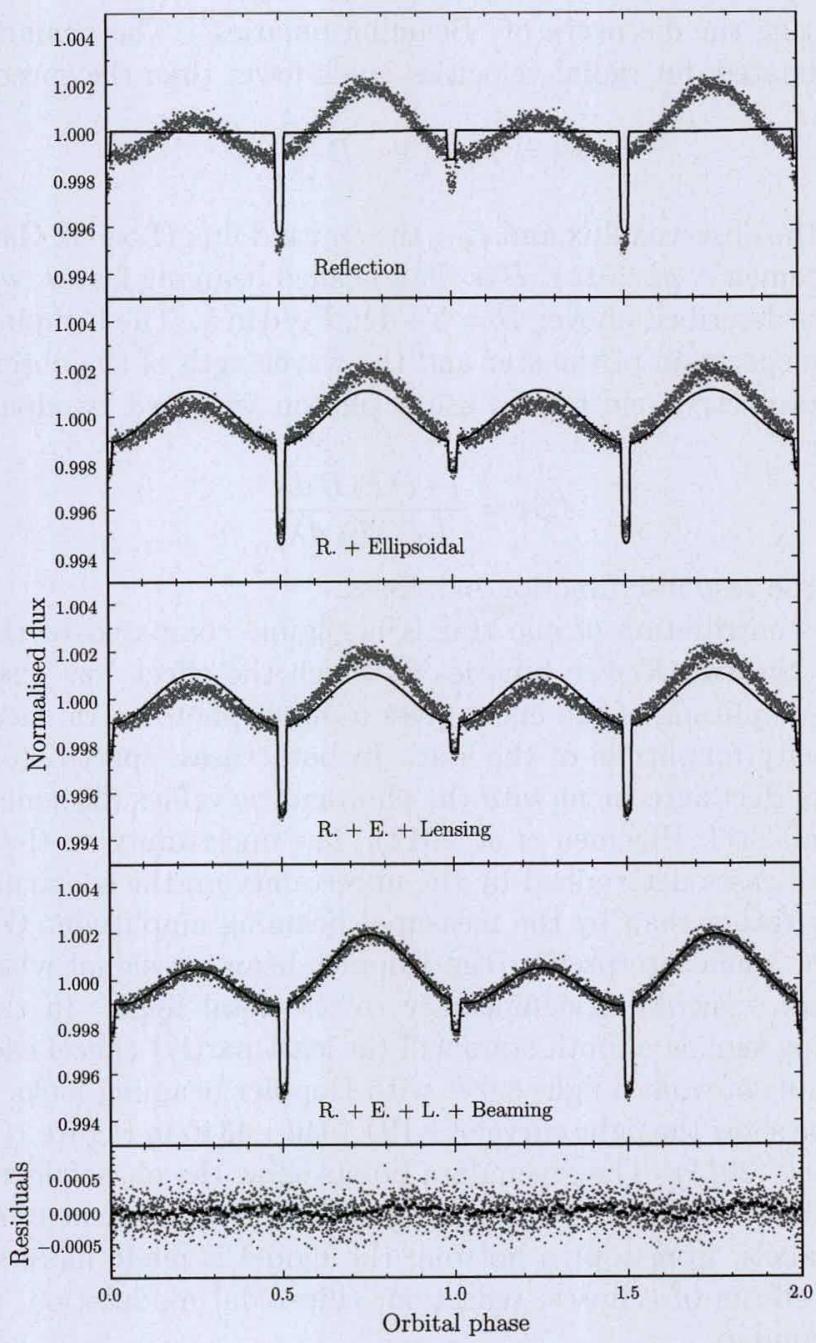

Fig. 1. Phase-folded, binned light curve of KPD 1946+4340 (green) and our best fitting model (black). In the top panel, only the eclipses and reflection effects are modelled. In the second panel, ellipsoidal modulation is added. In the third panel, gravitational lensing is taken into account as well, which affects the depth of the eclipse at orbital phase 0.5. The bottom panels show the full model - taking into account Doppler beaming and the residuals (grouped by 30 in green and grouped by 600 in black). Originally published in Bloemen et al. (2011).

periods (beaming and reflection go with the orbital period, ellipsoidal modulation at twice the orbital period), one can describe a light curve of a non-eclipsing binary with a circular orbit fairly well using such three sine curves, and one can 
even detect binaries using this triple-sine model ("BEER" model, see Shporer et al. 2011; Faigler \& Mazeh 2011; Faigler et al. 2012, 2013).

On a smaller-scale level, beaming of light by a rotating star leads to a photometric analogue of the Rossiter-McLaughlin effect (Rossiter 1924; McLaughlin 1924) that is seen in radial velocity measurements of eclipsing binaries. The part of the stellar surface that moves towards the observer due to the rotation of the star, appears slightly brighter than the part moving away. During eclipse, the eclipse depth will therefore be slightly enhanced when a blueshifted part of the stellar surface is eclipsed. Computations of how large this effect is are presented in Groot (2012), and Shporer et al. (2012) discuss the prospects of measuring spin-orbit misalignments using this signal.

A correct modelling of the Doppler beaming signal is not only required to correctly explain the light curves of certain binaries, it also allows one to get information about the radial velocity amplitudes of the stars, and therefore gives information that constrains the mass ratio of the system. The effect therefore has to be included in modelling codes. To model KPD $1946+4340$, the effect was implemented in LCURVE following the equations presented above. For wide filters, and stars that do not have strong spectral lines around the edges of the filters (which can then shift in or out of the bandpass), the linear approximation presented in 2 is very good.

\section{Light travel time effects}

Kepler has also allowed the characterisation of binaries and multiple systems through light travel time effects (also known as Rømer delays). These are apparent in eclipsing binaries that have a tertiary companion, where deviations from a constant period are seen in the observed eclipse timings. Such eclipse timing variations are discussed in Conroy et al. (2013) and Conroy et al. (this volume). Although eclipse timings are hard to measure reliably due to, e.g., spot modulations and pulsations, they carry enormous diagnostic information about the sizes of orbits and therefore masses of components of multiple-body systems.

Light travel time delays can also be observed in binary systems that do not have companions. A nice illustration is the effect of light travel time on the signal of a pulsating star in a binary orbit. An increasing light travel time when the star moves away from the observer, makes the pulsation periods appear longer. This is shown in Telting et al. (2012), where the light travel time across the orbit of a binary is measured using the signal of stellar oscillations from a subdwarf B star. The measured size of the orbit is in agreement with the velocities measured from spectroscopy as well as Doppler beaming. The light travel time effect can also be explained and modelled using a Doppler modulation of the pulsation frequencies, which makes it possible to also detect it using Fourier transforms (Shibahashi \& Kurtz 2012). Note that in the case of a binary in which both components show stellar pulsations, the detection of such frequency modulations in principle offer one the (so far unexploited) possibility to disentangle the pulsation spectra from the two stars. 
Another example of a method to detect the size of a binary orbit using light travel time delays is presented by Kaplan (2010): when a binary has a circular orbit and a mass ratio that is significantly different from unity, primary and secondary eclipses are in reality separated in time by half the orbital period, but the observed time difference is slightly different, because the stars that emit and block light are at a different distance from the observer during primary and secondary eclipse. A measure of this time difference, in combination with a measurement of the velocity of one of the stars through Doppler beaming, allows one to measure the mass ratio directly from photometric time series. The first detections of such time offsets where presented by Bloemen et al. (2012) for KOI-74 (56 $\pm 17 \mathrm{~s})$ and Barlow et al. (2012) for an $\mathrm{sdB}+\mathrm{M}$ dwarf binary $(2.06 \pm 0.12 \mathrm{~s})$. Very recently, Kaplan et al. (2013) reported the first detection using ground based data in Ultracam Dhillon et al. (2007) observations of NLTT 11748.

Thanks to all these recent examples of light travel time observations, it has become clear that light travel time has to be taken into account in modelling codes rather than assuming that all light that is emitted at one point in time reaches the other bodies in the system, and the observer, instantaneously.

\section{Lensing}

Several studies, e.g. Marsh (2001), showed that gravitational lensing can be important in eclipsing binary stars with a compact component. When the white dwarf eclipses its companion, light from the companion gets bent and amplified. As a result, the eclipse is a bit less deep. Sahu \& Gilliland (2003) showed that in a system with a typical white dwarf at 1 au of a main-sequence star, the lensing effect will swamp the eclipse signal completely. Although a typical white dwarf is Earth-sized, a transit of a white dwarf at 1 au of a star is therefore easily distinguishable from an Earth-like planet at the same distance from its host star.

The first times that lensing effects had to be taken into account when modelling the light curve, are the studies of the Kepler light curves of KPD 1946+4340 (Bloemen et al. 2011) and KOI-256 (Muirhead et al. 2013). In the case of KPD $1946+4340$ the eclipse depth is reduced by $\sim 12$ per cent, and as such, neglecting the lensing effect would lead to an underestimation of the white dwarf's radius by $\sim 6$ per cent.

\section{Conclusions and PHOEBE 2.0}

Thanks to the availability of superb light curves for hundred thousands of stars observed by the Kepler and CoRoT satellites, effects such as Doppler beaming, lensing, and light travel time delays have been seen in a growing number of eclipsing binaries. These effects allow a much better characterisation of binary stars, sometimes even significantly reducing the need for follow-up spectroscopy. In most widely used binary light curve modelling codes, such as the Wilson-Devinney code (see e.g., Wilson \& Devinney 1971; Wilson \& van Hamme 2010) and the PHOEBE 
package built on top of it (Prša \& Zwitter 2005), these effects are not yet included. Other, less generally applicable codes such as LCURVE have been adapted, but have important limitations, such as the need for circular orbits in the case of LCURVE. As explained in Prsa et al., this volume, we have therefore recently started developing PHOEBE 2.0, which includes the above effects but also offers higher flexibility in terms of systems (binaries, multiples, accretion discs, ...) and internal consistency, as well as improvements in surface discretization et cetera.

A nice example of the many improvements in PHOEBE 2.0 is the way Doppler beaming is implemented. The look-up tables that are used to get the flux of a surface element have been given an extra dimension (next to the standard ones such as wavelength/bandpass, effective temperature and surface gravity), which is the radial velocity. Rather than computing the flux and correcting for the Doppler beaming effect, the shift of the spectrum is now included in the tables, which is more accurate than using the linear approximation since it can also account for, for example, a spectral line that shifts in or out of the bandpass. Since in PHOEBE 2.0 every surface element has its own radial velocity value associated to it, which includes both orbital movement and stellar rotation, the photometric analogue of the Rossiter-McLaughlin effect is naturally included without adding further complexity to the model.

The new code is discussed in Degroote et al. (this volume), and multiple papers that are in preparation.

SB is supported by the Foundation for Fundamental Research on Matter (FOM), which is part of the Netherlands Organisation for Scientific Research (NWO).

The research leading to these results has received funding from the European Research Council under the European Community's Seventh Framework Programme (FP7/20072013)/ERC grant agreement No. 227224 (PROSPERITY), as well as from the Research Council of the KU Leuven under grant agreement GOA/2013/012.

\section{References}

Auvergne, M., Bodin, P., Boisnard, L., et al., 2009, A\&A, 506, 411

Barlow, B.N., Wade, R.A., Liss, \& S.E., 2012, ApJ, 753, 101

Bloemen, S., Marsh, T.R., Degroote, P., et al., 2012, MNRAS, 422, 2600

Bloemen, S., Marsh, T.R., Østensen, R.H., et al., 2011, MNRAS, 410, 1787

Borucki, W.J., Koch, D., Basri, G., et al., 2010, Science, 327, 977

Conroy, K.E., Prša A., Stassun, K.G., et al., 2013, AJ, in press [arXiv: 1306. 0512]

Copperwheat, C.M., Marsh, T.R., Dhillon, V.S., et al., 2010, MNRAS, 402, 1824

Dhillon, V.S., Marsh, T.R., Stevenson, M.J., et al., 2007, MNRAS, 378, 825

Ehrenreich, D., Lagrange, A., Bouchy, F., et al., 2011, A\&A, 525, A85

Faigler, S., \& Mazeh, T., 2011, MNRAS, 415, 3921

Faigler, S., Mazeh, T., Quinn, S.N., Latham, D.W., \& Tal-Or, L., 2012, ApJ, 746, 185

Faigler, S., Tal-Or, L., Mazeh, T., Latham, D.W., \& Buchhave, L.A., 2013, ApJ, 771, 26

Groot, P.J., 2012, ApJ, 745, 55 
Kaplan, D.L., 2010, ApJ, 717, L108

Kaplan, D.L., Marsh, T.R., Walker, A.N., et al., 2013, ApJ, in press [arXiv:1311.6831]

Loeb, A., \& Gaudi, B.S., 2003, ApJ, 588, L117

Marsh, T.R., 2001, MNRAS, 324, 547

Maxted, P.F.L., Marsh, T.R., \& North, R.C., 2000, MNRAS, 317, L41

McLaughlin, D.B., 1924, ApJ, 60, 22

Muirhead, P.S., Vanderburg, A., Shporer, A., et al., 2013, ApJ, 767, 111

Prša, A., Batalha, N., Slawson, R.W., et al., 2011, AJ, 141, 83

Prša, A., \& Zwitter, T., 2005, ApJ, 628, 426

Rossiter, R.A., 1924, ApJ, 60, 15

Sahu, K.C., \& Gilliland, R.L., 2003, ApJ, 584, 1042

Shibahashi, H., \& Kurtz, D.W., 2012, MNRAS, 422, 738

Shporer, A., Brown, T., Mazeh, T., \& Zucker, S., 2012, NewA, 17, 309

Shporer, A., Jenkins, J.M., Rowe, J.F., et al., 2011, AJ, 142, 195

Slawson, R.W., Prša, A., Welsh, W.F., et al., 2011, AJ, 142, 160

Telting, J.H., Østensen, R.H., Baran, A.S., et al., 2012, A\&A, 544, A1

van Kerkwijk, M.H., Rappaport, S.A., Breton, R.P., et al., 2010, ApJ, 715, 51

Wilson, R.E., \& Devinney, E.J., 1971, ApJ, 166, 605

Wilson, R.E., \& van Hamme, W., 2010, in Binaries - Key to Comprehension of the Universe, ASP Conf. Ser., 435, 45

Zucker, S., Mazeh, T., \& Alexander, T., 2007, ApJ, 670, 1326 
Copyright of EAS Publications Series is the property of Cambridge University Press and its content may not be copied or emailed to multiple sites or posted to a listserv without the copyright holder's express written permission. However, users may print, download, or email articles for individual use. 